\title{
EVALUACIÓN DE TECNOLOGÍAS SANITARIAS: LA EXPERIENCIA EN EL MINISTERIO DE SALUD DE BRASIL
}

\author{
Marcus Tolentino Silva $a^{1,2, a}$
}

\begin{abstract}
RESUMEN
El presente artículo tiene como objetivo describir las principales actividades llevadas a cabo por el Ministerio de Salud de Brasil (MINSAB) para el desarrollo del área de Evaluación de Tecnologías Sanitarias (ETS). Se trata de una descripción basada en los esfuerzos y estrategias del MINSAB para fortalecer esta área en los últimos años. Son cinco los ejes de acción acogidos para dicho propósito: (i) la adopción de una Política Nacional para la Gestión de Tecnologías en Salud; (ii) el apoyo al proceso de toma de decisiones en el Sistema Único de Salud; (iii) la promoción de la realización de estudios en ETS; (iv) la formación de recursos humanos estratégicos y (v) la articulación institucional nacional e internacional. No obstante, persisten los desafíos para lograr una estructuración más efectiva de la ETS en Brasil, como la creación de una institución gubernamental con mayor agilidad administrativa, entre otras.
\end{abstract}

Palabras clave: Evaluación en Salud; Estudios de Evaluación como Asunto; Evaluación de tecnologías sanitarias; Economía de la Salud; Sistema único de salud; Brasil (fuente: DeCS BIREME).

\section{HEALTH TECHNOLOGY ASSESSMENT: EXPERIENCE IN THE MINISTRY OF HEALTH OF BRAZIL}

\begin{abstract}
This article aims to describe the main activities carried out by the Ministry of Health of Brazil (MHB) for the development of Health Technology Assessments (HTA). It is a description based on the MHB efforts and strategies to strengthen this area in the last years. Five are the lines of action put in place for that purpose: (i) adoption of a National Policy for the Management of Health Technology, (ii) supporting the decision making process in the Unified Health System, (iii ) promotion of the performance of studies on HTA, (iv) training of strategic human resources, and (v) institutional coordination at national and international levels. However, challenges remain to achieve a more effective structure for HTA in Brazil, like the creation of a government institution with greater administrative flexibility, among others.
\end{abstract}

Key words: Health Evaluation; Evaluation Studies as Topic; Health Technology Assessment; Health Economics; Unified Health System; Brasil (source: MeSH NLM).

\section{INTRODUCCIÓN}

Las actividades relacionadas con la evaluación de tecnologías surgieron a mediados de los años 60 , como uno de los mecanismos para identificar las consecuencias indirectas de las tecnologías en la sociedad moderna. La experiencia de los efectos observados en procesos químicos, industriales y agrícolas, reforzó la necesidad de tales acciones. Con este objetivo, la evaluación de tecnologías pretende medir el impacto deseado - y no deseado - de las tecnologías en la sociedad, la economía y el medio ambiente ${ }^{(1)}$.

Específicamente en el sector salud, estas evaluaciones pretenden ayudar en el proceso de toma de decisiones relacionadas con las tecnologías en salud (2). Las tecnologías en salud incluyen medicamentos, materiales, equipamientos y procedimientos, sistemas organizacionales, educacionales, de información y de apoyo, programas y protocolos asistenciales, a través de los cuales se brinda atención y cuidado a la salud de la población ${ }^{(3)}$.

En los últimos años, además de cambiar el perfil epidemiológico de la población, se ha observado un crecimiento continuo de los gastos en salud en forma paralela a la producción de nuevas tecnologías y avances en el conocimiento científico. En Brasil, este hecho ha tenido un impacto significativo en la sostenibilidad del Sistema Único de Salud (SUS) ${ }^{(3)}$. Este incremento de la oferta, no necesariamente incrementó los beneficios que la sociedad podría haber tenido, y pueda aún tener,

Departamento de Ciência e Tecnologia, Secretaria de Ciência, Tecnologia e Insumos Estratégicos, Ministério da Saúde. Brasilia DF, Brasil.

Núcleo de Saúde Baseada em Evidências e Comunicação Científica, Faculdade de Ciencias da Saúde, Universidad de Brasilia. Brasilia DF, Brasil.

Farmacéutico, Magíster en Efectividad en Salud Basada en Evidencias, Especialista en Epidemiología

$$
\text { Recibido: 15-07-11 Aprobado: 24-08-11 }
$$


con la utilización racional de estas tecnologías. Parte del problema se refiere a la incorporación de tecnologías que aportan beneficios nulos, inciertos o perjudiciales para la salud de la población (1).

La Evaluación de Tecnologías Sanitarias (ETS) es el proceso continuo de análisis y síntesis de los beneficios para la salud, de las consecuencias económicas y sociales del uso de las tecnologías, teniendo en cuenta los siguientes aspectos: seguridad, precisión, eficiencia, eficacia, efectividad, costos, costo-efectividad y aspectos de equidad, impactos éticos, culturales y ambientales involucrados en su uso ${ }^{(3)}$. Estas dimensiones requieren un enfoque multidisciplinario para el análisis, que abarca diversas disciplinas, tales como la epidemiología, estadística, economía, ingeniería, medicina, entre otras ${ }^{(1)}$.

En Brasil, las iniciativas de ETS comenzaron en grupos de investigación e instituciones académicas ${ }^{(4)}$. En los últimos años, el Ministerio de Salud (MINSAB) subvencionó acciones gubernamentales para el fortalecimiento de las ETS en el SUS, encaminadas a consolidar una Red Brasileña de Evaluación de Tecnologías en Salud ${ }^{(5)}$.

El modelo adoptado por el MINSAB refleja la tendencia internacional, con respecto a las características específicas del SUS y el modelo de enseñanza, investigación y desarrollo vigente en el país. Con el objetivo de orientar los procesos de toma de decisiones relacionadas con las tecnologías en salud en el SUS, el MINSAB priorizó cinco estrategias, las cuales se presentan de modo sucinto en el presente artículo.

\section{ADOPCIÓN DE UNA POLÍTICA NACIONAL DE GESTIÓN DE TECNOLOGÍAS EN SALUD}

La Política Nacional de Gestión de Tecnologías en Salud (PNGTS) es el instrumento rector para los actores involucrados en la gestión de los procesos de evaluación, adquisición, difusión, gestión de la utilización y retiro de tecnologías en el SUS. Bajo la supervisión del MINSAB, la PNGTS es el resultado de las discusiones entre 2005 a 2009 entre los distintos sectores de la sociedad. La PNGTS tiene como objetivo maximizar los beneficios para la salud que se derivan de los recursos disponibles, garantizando el acceso de la población a tecnologías seguras y efectivas, en términos de equidad (3).

La PNGTS tiene las siguientes directrices:

- Utilizar evidencias científicas para apoyar la gestión a través de la evaluación de tecnologías en salud;

- Mejorar el proceso de incorporación de tecnologías;
- Racionalizar el uso de las tecnologías;

- Apoyar el fortalecimiento de la enseñanza y la investigación en la gestión de tecnologías en salud;

- Sistematizar y difundir la información;

- Fortalecer las estructuras gubernamentales; y

- Fomentar la articulación político-institucional e intersectorial $^{(3)}$.

La adopción de estas directrices, en la perspectiva del MINSAB, se inició incluso antes de su institucionalización. Desde el 2006 fueron instituidos procesos de evaluación y de incorporación de tecnologías basados en el análisis de las consecuencias y de los costos para el sistema de salud y para la población (4). Como se describe a continuación, las demás estrategias tienen la PNGTS como guía, y pretenden sensibilizar a la sociedad brasileña de la importancia de las consecuencias económicas y sociales del uso inadecuado de las tecnologías en los servicios de salud del SUS.

\section{APOYO EN EL PROCESO DE TOMA DE DECISIONES EN EL SISTEMA ÚNICO DE SALUD}

La organización y funcionamiento del SUS fue descrito en un artículo previo ${ }^{(6)}$. Con respecto a la necesidad de la toma de decisiones sobre el acceso a las tecnologías en salud, todos los niveles del sistema están involucrados, es decir, el nivel Federal (Nacional), Estatal (Estados) y municipal (Municipios). Debido a las numerosas posibilidades de análisis relacionados con tecnologías en salud - desde el levantamiento inmediato de las mejores evidencias de eficacia y seguridad disponible hasta un estudio de costo-efectividad o costo-utilidad - el MINSAB adoptó un modelo que distingue la producción de ETS en dos vertientes: (1) una de producción interna (elaborada por el cuerpo técnico) y (2) otra de producción externa, a través del apoyo a las instituciones educativas y de investigación.

En resumen, la producción interna consiste en la elaboración de notas técnicas de revisión rápida (elaboradas en una o dos semanas) y de informes técnico-científicos (elaborados hasta en tres meses). Esta producción tiene como objetivo resumir la mejor evidencia disponible sobre el uso de determinada tecnología para una condición específica, con el fin de ayudar a los gerentes del SUS en cualquier toma de decisión que demanda una mayor velocidad administrativa.

Con el objetivo de mejorar la calidad de los informes técnico-científicos preparados, el MINSAB elaboró una norma metodológica ${ }^{(7)}$. Después de su publicación, las 
normas fueron utilizadas en diversos contextos en todo Brasil y en los tres niveles del SUS, así como, en la Salud Suplementaria (planes privados de salud). La experiencia del MINSAB permitió el establecimiento de un flujo de producción de informes técnico-científicos, que incluye la elaboración por parte de los asesores técnicos, la revisión metodológica de la búsqueda y análisis crítico de los estudios seleccionados y la revisión de especialistas y de los gerentes involucrados con las políticas de atención a la salud. Además, el MINSAB ofreció talleres de capacitación para la elaboración de informes técnico-científicos, con la finalidad de capacitar personal con efecto multiplicador en los diferentes niveles del sistema.

\section{FOMENTO A LA REALIZACIÓN DE ESTUDIOS EN LA EVALUACIÓN DE TECNOLOGÍAS EN SALUD}

La producción externa consiste en apoyar el desarrollo de estudios que tengan la temática de las ETS, como las revisiones sistemáticas, evaluaciones económicas, análisis de la gestión de tecnología en salud, entre otros. En promedio, estos estudios se realizan en un máximo de dos años, incluyen análisis más complejos, recogen información no disponible en la rutina habitual y pueden apoyar a la producción interna en el futuro.

Particularmente en Brasil, a nivel gerencial se presentan serias dificultades para aceptar resultados de investigaciones realizadas en otros contextos, y que sean de difícil asimilación. Además, el gerente no tiene la capacidad (o personal técnico calificado) para identificar los potenciales sesgos de publicación y de evaluar críticamente la información científica ${ }^{(4,8)}$.

De modo más operativo, la producción externa realizada por el MINSAB en la ETS, se consolidó en los últimos años en cinco etapas: (i) identificación; (ii) establecimiento de prioridades; (iii) promoción; (iv) monitoreo; y (v) difusión. Estas actividades tuvieron como objetivo el acercamiento a las necesidades de gestión del SUS con estudios apoyados mediante la integración presencial de gerentes e investigadores en todas las fases de la investigación (desde el diseño del proyecto hasta la difusión de los resultados).

La participación de diversos actores en este proceso incrementó la complejidad de las etapas. Además, junto a otro conjunto de actividades, esta acción difundió, aunque modestamente, la cultura de la ETS por los formuladores de políticas y los encargados de la toma de decisiones del SUS. Se ha detectado el uso de términos propios de la ETS en los discursos y notas de representantes del MINSAB ${ }^{(2,4)}$.
EI MINSAB tiene diversas estrategias para promover estudios en la ETS. La estrategia que abarca el mayor volumen de recursos es la organización de convocatorias públicas, en colaboración con el Ministerio de Ciencia y Tecnología. El concurso público de proyectos permite fortalecer las iniciativas académicas emergentes y la consecuente formación de experiencia en el área.

\section{FORMACIÓN DE RECURSOS HUMANOS ESTRATÉGICOS}

Desde la época de los debates de las Política Nacional de Gestión de Tecnologías en Salud, el MINSAB incentivó la formación de recursos humanos en diversas modalidades y áreas del conocimiento involucradas en las fases del ciclo de vida de las tecnologías. Vale la pena destacar el apoyo a los cursos de postgrado (especialización, maestría y postdoctorado) y educación continua (por videoconferencia o presencial) para los gestores y profesionales de los servicios de salud, especialmente en el campo de la ETS y de la economía de la salud.

La primera iniciativa para la estructuración de la Red Brasileña de ETS fue el establecimiento de alianzas con instituciones académicas para la realización de cursos de postgrado en gestión de tecnologías en salud dirigidas a los profesionales del SUS ${ }^{(5)}$.

\section{ARTICULACIÓN INSTITUCIONAL NACIONAL E INTERNACIONAL}

La mayor parte del crecimiento de la ETS en el SUS es el resultado de la articulación institucional en el ámbito federal de todos los sectores relacionados con la gestión de tecnologías en salud. Esta articulación permitió aprobar con diversas instancias las estrategias anteriormente descritas, incluida la formulación de la PNGTS. Actualmente, en el país existen diversos foros de debate relacionados a la ETS, vinculadas o no al SUS, en la que tiene base el MINSAB.

Entre los diversos foros nacionales, se destaca la Red Brasileña de ETS (REBRATS, de sus siglas en portugués), que reúne a sus integrantes con un objetivo común: promover y difundir el área de la ETS en Brasil. Actúa como un puente entre la investigación y la gestión, concediendo subvenciones para decisiones de incorporación, monitoreo y abandono de las tecnologías en el contexto de sus usos en el sistema de salud. También es una herramienta estratégica para facilitar la estandarización de metodologías, validar y certificar la calidad de los estudios y establecer una educación continua ${ }^{(5)}$. 
En cuanto a la inserción internacional del MINSAB en el área de la ETS, se destaca su participación activa en el foro del MERCOSUR ${ }^{(9)}$ y como institución miembro de la Red Internacional de Agencias de Evaluación de Tecnologías en Salud (International Network of Agencies for Health Technology Assessment- INHATA) ${ }^{(10)}$. Coronando el conjunto de iniciativas, Brasil fue sede de la octava reunión de la Sociedad Internacional de Evaluación de Tecnología en Salud (Health Technology Assessment International-HTAi) ${ }^{(11)}$.

\section{COMENTARIOS FINALES}

El cambio constante de las etapas de difusión de las tecnologías en salud induce a los formuladores de políticas y tomadores de decisiones a apoyar de modo permanente las actividades de la ETS. Para minimizar la duplicidad de acciones se priorizan los estudios dirigidos a la evaluación crítica y síntesis de la evidencia disponible, como las revisiones sistemáticas. No obstante, la información científica de métodos diagnósticos y terapéuticos proviene frecuentemente de países desarrollados que adoptan sus parámetros epidemiológicos y de su capacidad instalada, que no necesariamente puede reflejar el contexto brasileño.

Al igual que ocurre en otros países, el mayor desafío de la ETS en Brasil radica en transformarla en una herramienta para priorizar acciones en salud pública y no en un mecanismo de respuesta a las presiones del mercado.

La adopción de la ETS por el MINSAB es fundamental para apoyar las instancias de decisiones relativas a la incorporación de tecnologías en el SUS, ya que existe una presión organizada y sectorizada de la población. Por otro lado, las actividades de la ETS no disminuirán las desigualdades en salud, ya que la población de mayor riesgo no consigue posicionarse políticamente y, por lo tanto, no articula sus prioridades.

Considerando el crecimiento exponencial de la aparición de nuevos conocimientos y los modelos adoptados en otros países, es imperativo que sea establecida una institución con mayor velocidad administrativa, para minimizar los riesgos de expiración de análisis frente a una necesidad de la gestión del SUS.

A pesar que la síntesis de los parámetros económicos anotados en la definición corrobore las consideraciones sobre la equidad del acceso a las tecnologías en salud, es crucial que la ETS considere en su análisis el impacto de la sostenibilidad del SUS. Esto implica conocer la capacidad de ofertar bienes y prestar servicios de acuerdo a las necesidades de salud de la generación presente sin comprometer la oferta de bienes y la prestación de servicios necesarios para las generaciones futuras.

\section{Conflictos de interés}

El autor trabaja en el Ministerio de Salud de Brasil, en el área de evaluación de tecnologías en salud desde mayo de 2005

\section{REFERENCIAS BIBLIOGRÁFICAS}

1. Goodman CS. HTA 101: introduction to health technology assessment. Bethesda, MD: National Library of Medicine; 2004.

2. Bueno RLP. A decisão de incorporação de tecnologias em saúde no SUS: a questão dos medicamentos [Tesis doctoral]. Porto Alegre: Universidade Federal do Rio Grande do Sul, Escola de Administração; 2010.

3. Ministério da Saúde da Brasil. Política Nacional de Gestão de Tecnologias em Saúde. Brasilia, Brasil: Ministério da Saúde; 2010. Disponible en: http://bvsms.saude.gov.br/bvs/publicacoes/politica_nacional_gestao_tecnologias_saude.pdf

4. Nita ME, Secoli SR, Nobre MRC, Ono-Nita SK, Campino ACC, Sarti FM, et al. Avaliação de tecnologias em saúde: evidência clínica, análise econômica e análise de decisão. Porto Alegre: Artmed, 2010

5. Rede Brasileira de Avaliação de Tecnologias em Saúde, Ministério de Saúde da Brasil [Internet]. Brasilia, Brasil: Ministerio de Salud;2010 [citado el 20 de julio 2011]. Disponible en: www.saude.gov.br/rebrats

6. Silva MT. Sistema Único de Salud: la experiencia brasileña en la universalización del acceso a la salud. Rev Peru Med Exp Salud Publica. 2009;26(2):251-7.

7. Ministério da Saúde da Brasil. Diretrizes metodológicas: elaboração de pareceres técnico-científicos. Brasilia, Brasil: Ministério da Saúde; 2010. Disponible en: http://200.214.130.94/rebrats/publicacoes/ptc_2009.pdf

8. Ministerio da Saúde da Brasil. Avaliação de Tecnologias em Saúde Ferramentas para a Gestão do SUS. Brasilia, Brasil: Ministério da Saúde; 2009. Disponible en: http://bvsms. saude.gov.br/bvs/publicacoes/avaliacao_tecnologias_saude_ferramentas_gestao.pdf

9. MERCOSUR [Internet]. Montevideo, República Oriental del Uruguay; 2010 [citado el 20 de julio 2011]. Disponible en:www.mercosur.int

10. International Network of Agencies for Health Technology Assessment [Internet]. Estocolmo, Suecia; 2011 [citado el 20 de julio 2011]. Disponible en: www.inahta.org

11. Health Technology Assessment Society [Internet]. Rio de Janeiro, Brasil; 2011 [citado el 20 de julio 2011]. Disponible en: www.htai2011.org

Correspondencia: Marcus Tolentino Silva

Dirección: Esplanada dos Ministérios, Bloco G, Edifício Sede, sala 852. Brasilia DF, Brasil.

Correo electrónico: marcus.silva@saude.gov.br 